\title{
Perioperative care with an ultrasound device is as Michael Jordan with Scotty Pippen: at its best!
}

\author{
Jip Nooitgedacht ${ }^{1}$, Mark Haaksma ${ }^{1}$, Hugo R. W. Touw ${ }^{2}$, Pieter R. Tuinman ${ }^{1,3}$ \\ ${ }^{1}$ Department of Intensive Care Medicine, Amsterdam UMC, Vrije Universiteit Amsterdam, Amsterdam, The Netherlands; ${ }^{2}$ Department of Intensive \\ Care Medicine, Radboud University Medical Centre, Nijmegen, The Netherlands; ${ }^{3}$ Amsterdam Leiden Intensive Care Focused Echography (ALIFE) \\ Correspondence to: Pieter Roel Tuinman, MD, PhD. Department of Intensive Care Medicine, Amsterdam UMC, Vrije Universiteit Amsterdam, \\ Amsterdam, The Netherlands. Email: p.tuinman@vumc.nl. \\ Provenance: This is an invited Editorial commissioned by the Section Editor Shuangjiang Li (Department of Thoracic Surgery and West China \\ Medical Center, West China Hospital, Sichuan University, Chengdu, China). \\ Comment on: Chiappetta M, Meacci E, Cesario A, et al. Postoperative chest ultrasound findings and effectiveness after thoracic surgery: A pilot study. \\ Ultrasound Med Biol 2018;44:1960-7.
}

Submitted Nov 29, 2018. Accepted for publication Dec 13, 2018.

doi: $10.21037 /$ jtd.2018.12.82

View this article at: http://dx.doi.org/10.21037/jtd.2018.12.82

Perhaps one of the greatest success stories in the diagnostic approach of dyspneic patients is the introduction of lung ultrasound (LUS). In the past, LUS was considered impossible due to full reflection of the ultrasound beam by air. However, we now know that the distinctive artifacts created by the interplay of air, fluid and pleurae are of great diagnostic value and can help differentiate between various pathological processes (1).

Ever since the introduction of LUS by the landmark study by Lichtenstein et al. (2), the use of LUS, and other forms of point-of-care ultrasound (POCUS), as diagnostic tool in the emergency setting has rapidly expanded. In addition, a recent meta-analysis on studies in critically ill patients showed that LUS estimates of sensitivity (95\%) and specificity (94\%) were much higher than the estimates of chest radiograph (CXR) (sensitivity $49 \%$ and specificity $92 \%$ ) when compared with computed tomography (CT) as reference standard (3). Other advantages of LUS are being non-invasive, cost and time effective, and 'bedside' available, whereas CXR and CT come with a cost, radiation, contrast and/or transportation burden. Despite LUS being superior in accuracy and feasibility, CXR is still considered the firstline diagnostic imaging modality for almost all patients presenting with pulmonary pathology in different settings.

Apart from the use of LUS at the bedside of the acute dyspneic patient, other applications of POCUS are rapidly gaining ground. POCUS has proven its value as a periprocedural tool, e.g., during real time guidance of central venous catheterization and thoracentesis (4-6). Also, the use of POCUS in evaluating diaphragm functionality is of interest. Research has shown that the use of POCUS to measure diaphragm thickness, motion and thickening fraction can quantify diaphragm atrophy and predict weaning success $(7,8)$.

Regarding LUS, when taking the foregoing into account, it is a logical next step to study its application in perioperative care. Patients after cardiothoracic surgery are of special interest, since postoperative pulmonary complications are common in these patients and associated with adverse outcomes (9). Although the use of LUS in perioperative care seems like common-sense, still limited evidence of its value in perioperative care is available.

However, since recent years, studies regarding the use of LUS in identifying postoperative pulmonary complications after cardiothoracic surgery are emerging (Table 1), which we will summarize here.

A prospective cohort trial by Goudie et al., included patients admitted to the recovery ward following thoracic surgery (10). Patients were evaluated by LUS and compared to CXR as a reference standard. The presence of pneumothorax and/or pleural effusion was studied. With a sensitivity and specificity of $83 \%$ and $59 \%$ for pleural effusions and $21 \%$ and $95 \%$ for pneumothorax respectively, the authors concluded LUS may be able to reduce the 
Table 1 Overview of current research on the use of chest point-of-care ultrasound, e.g., lung ultrasound and TTE, in perioperative care of cardiothoracic patients

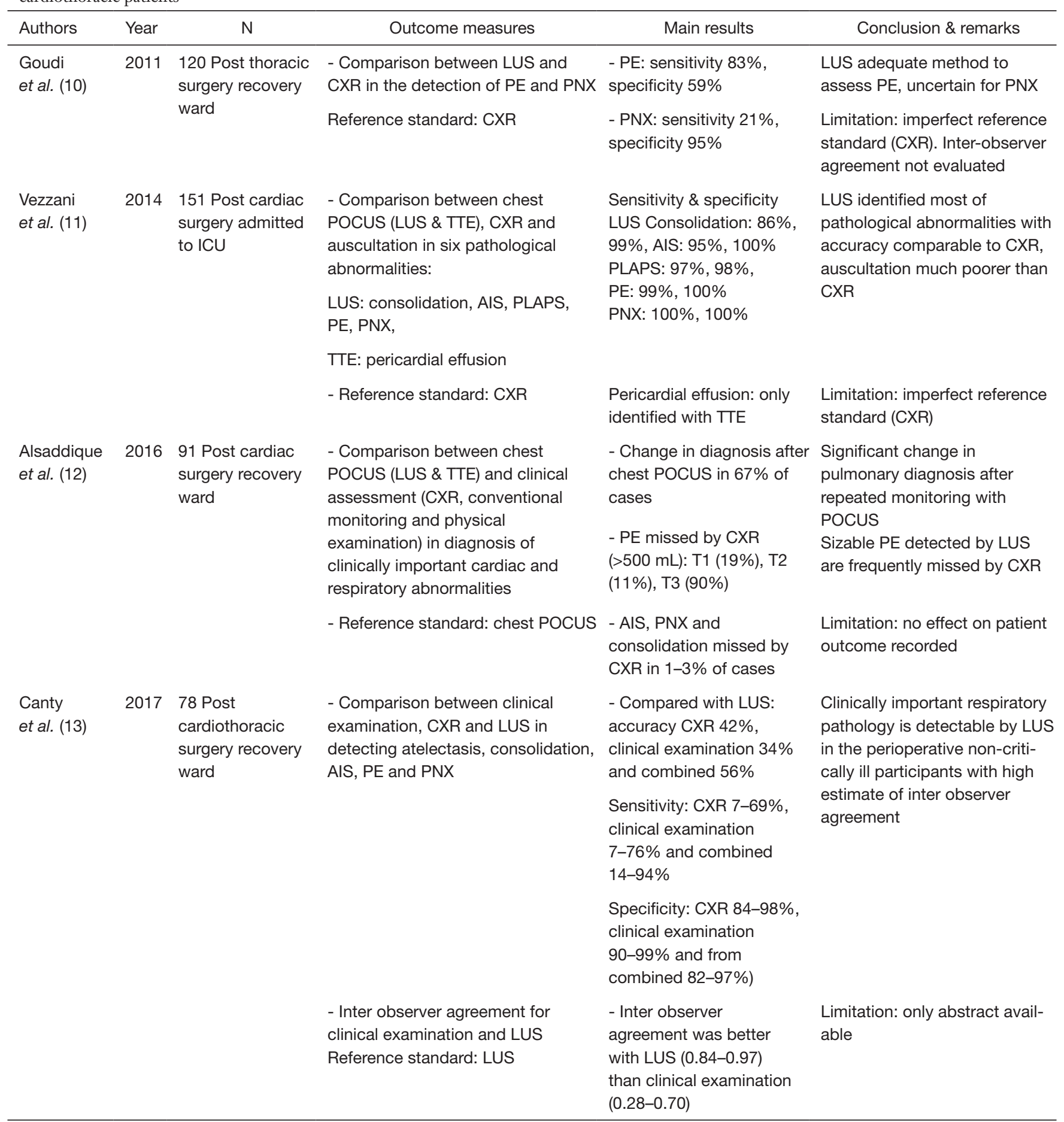

Table 1 (continued) 
Table 1 (continued)

\begin{tabular}{|c|c|c|c|c|c|}
\hline Authors & Year & $\mathrm{N}$ & Outcome measures & Main results & Conclusion \& remarks \\
\hline \multirow{2}{*}{$\begin{array}{l}\text { Touw } \\
\text { et al. (14) }\end{array}$} & & & $\begin{array}{l}\text { - Comparison between LUS and } \\
\text { CXR in time to detection clinically } \\
\text { relevant PPCs }\end{array}$ & $\begin{array}{l}\text { - Clinically relevant PPCs: } \\
65 \% \text { and } 41 \% \text { were } \\
\text { detected by LUS and CXR, } \\
\text { respectively }(P<0.001)\end{array}$ & $\begin{array}{l}\text { Limitation: use of composite } \\
\text { reference standard might } \\
\text { complicate replication and } \\
\text { comparison across studies }\end{array}$ \\
\hline & & & $\begin{array}{l}\text { - Reference standard: Clinically } \\
\text { relevant PPC's }\end{array}$ & $\begin{array}{l}\text { - Time to detection of } \\
\text { clinically relevant PPC: } \\
\text { LUS faster than CXR } \\
(P=0.024)\end{array}$ & \\
\hline \multirow{3}{*}{$\begin{array}{l}\text { Chiappetta } \\
\text { et al. (15) }\end{array}$} & & & $\begin{array}{l}\text { - Feasibility, limitation and clinical } \\
\text { value assessments }\end{array}$ & - Excellent feasibility report & $\begin{array}{l}\text { Limitation: small sample size, } \\
\text { no reference standard }\end{array}$ \\
\hline & & & - Reference standard: none & $\begin{array}{l}\text { - LUS limited by massive } \\
\text { SE and in most open } \\
\text { surgery cases }\end{array}$ & \\
\hline & & & & $\begin{array}{l}\text { - Clinical value: LUS } \\
\text { sufficient in detection PNX } \\
\text { and parenchymal } \\
\text { alterations in absence of } \\
\text { SE }\end{array}$ & \\
\hline
\end{tabular}

AIS, alveolar interstitial syndrome; CRS, composite reference standard; CXR, chest X-ray; ICU, intensive care unit; LUS, lung ultrasound; PE, pleural effusion; PLAPS, posterolateral alveolar and/or pleural syndrome; PNX, pneumothorax; PPC, postoperative pulmonary complication; TTE, transthoracic echocardiography; SE, subcutaneous emphysema.

number of routine CXR. They also found that certain findings of LUS and CXR were complementary to each other in diagnosing postoperative pulmonary complications, raising the question if using both techniques as respectively the primary and secondary diagnostic imaging modality could have additional value. Of note the values of LUS sensitivity and specificity, are very difficult to interpret, since CXR is a far from perfect gold standard and the accuracy of LUS (the index test) is probably greater than that of CXR (the reference test in this study).

A prospective observational study by Vezzani et al., included patients admitted to the ICU following cardiac surgery (11). Patients were evaluated by chest POCUS, auscultation and CXR, with CXR being the reference standard. Chest POCUS consisted of LUS and transthoracic echocardiography (TTE). The presence of consolidation, alveolar-interstitial syndrome, posterolateral alveolar and/or pleural syndrome (PLAPS), pleural effusion, pneumothorax and pericardial effusion was studied. Sensitivities and specificities of chest POCUS were comparable to those of CXR for these six different pathological findings. Sensitivity and specificity measures of lung auscultation were very low. Not surprisingly, TTE detected all pericardial effusions while neither CXR nor chest auscultation were able to identify them. Since this study also chose CXR as a reference standard, results regarding LUS accuracy are again difficult to interpret.

A prospective observational study by Alsaddique et al., 
included patients admitted to the recovery ward following cardiac surgery (12). Patients were evaluated by chest POCUS, CXR, conventional monitoring and physical examination with chest POCUS being the reference standard. Chest POCUS consisted of LUS and TTE, and was performed at three time points (day after surgery, after extubation and at discharge). A clinical diagnosis was made based on conventional monitors, CXR and physical assessment. The use of chest POCUS lead to a change in the initial clinical diagnosis in $67 \%$ of patients, where in particular LUS detected new pleural effusions or excluded suspected pleural effusions in respectively $33 \%$ and $14 \%$ of the patients.

A prospective observational study by Canty et al., included patients admitted to the recovery ward following cardiothoracic surgery (13). Patients were evaluated by LUS, CXR and clinical examination with chest POCUS being the reference standard. LUS was performed after clinical examination. Incidence of five different lung pathologies (atelectasis, consolidation, alveolar-interstitial syndrome, pleural effusion and pneumothorax) were assessed and compared between LUS, CXR and clinical examination. Compared with LUS, agreement in diagnosis of the five lung pathologies was poor. They concluded that clinically important respiratory pathology is detectable by LUS, where clinically significant diagnoses may be missed by clinical examination and CXR.

The largest prospective observational study by Touw et al., included patients admitted to the ICU after cardiothoracic surgery (14). Patients were evaluated by LUS and CXR with clinically relevant post pulmonary complications as composite reference standard. LUS was performed immediately before or after CXR on the day of surgery, and postoperatively on days two and three. Results showed that LUS detected more post pulmonary complications when compared to CXR, more clinically relevant complications, and importantly, at an earlier point in time and with excellent inter-observer agreement.

Recently, in Ultrasound in Medicine and Biology (2018), Chiappetta et al. reported the results of a prospective observational pilot study involving 24 patients who underwent thoracic surgery of various degrees of complexity (15). They compared LUS findings with CXR findings to evaluate if LUS alone might be sufficient for the evaluation of pulmonary complications after thoracic surgery, or the aid of CXR is still needed. They evaluated the exhaustiveness of LUS with the help of a questionnaire in which the examiners where asked if LUS was sufficient to diagnose chest pathology or CXR was needed. Interestingly, they gathered their findings per type of thoracic surgery with the aim to discriminate the viability of LUS in different patient populations. Also, they investigated the overall usability of the examination in terms of feasibility, limitations and clinical value.

Results showed that in the large majority of cases (79\%) LUS was sufficient and no CXR was needed for further clarification of complications. Their findings also suggested that LUS excels in detecting complications after minimally invasive thoracic surgery ( $85 \%$ of cases), and was thorough in detecting complications after open thoracic surgery (67\% of cases). In those cases where LUS was not sufficient and CXR had to be performed for clarification (21\%) subcutaneous emphysema was present or no lung point was found. In terms of clinical value, LUS detected five cases of pneumothorax and four of pleural effusion that were missed by CXR, but all were considered clinically insignificant. In addition, CXR detected six cases of parenchymal alterations, 2 of which LUS missed due to subcutaneous emphysema, pneumothorax and air leak, and four that were considered clinically insignificant. LUS was found feasible in terms of patient comfort, repeatability and time efficiency, and deemed suitable for routine examinations.

To our knowledge, this study is the first to evaluate LUS as a primary diagnostic tool and CXR as a complementary tool based on operator evaluation with the help of a questionnaire. This is an innovative way to study the exhaustiveness of LUS in the perioperative setting. Furthermore, their findings on subcutaneous emphysema confirm that, because a natural barrier is formed due to acoustic impedance between aqueous tissue and migrated air, that occludes the scattering of ultrasound signals, the composition of an ultrasound image is impossible. This is in concordance with the literature, yet they also showed that in cases with only limited amounts of subcutaneous emphysema, this problem can easily be solved by adjusting probe position $(16,17)$.

The conduct of this study reflects the considerable difficulties researchers face when comparing different imaging techniques in postoperative patients, as in the ideal situation a chest CT scan is used as reference standard, being the current gold standard. However, CT-scanning in these patients comes with a lot of downsides and is therefore understandably not used. In the aforementioned study by Touw et al., this issue was circumvented by using clinically relevant complications as a composite reference standard to minimize reference standard bias, which could 
serve as an example for future research (14). As the sample size of the pilot study of Chiappetta et al. was small, caution is warranted when interpreting the results, yet it also motivates further investigation, as already planned by the authors.

With this increasing evidence on the role of LUS in perioperative medicine, we are one step closer in defining its definite role in these patients. It seems clear that LUS can serve as the primary diagnostic tool, simply because it is more accurate than CXR (and auscultation), and provides more meaningful information

In order to integrate LUS (and POCUS) into daily routine practice, several steps must be taken to optimize and accelerate this process. First, it needs to be considered as the fifth pillar of clinical examination next to inspection, palpation, percussion and auscultation. Even more, with the development of handheld devices, LUS can extensively complement, and for many indications replace lung percussion and auscultation.

Second, ultrasound training must expand to other specializations outside intensive care and emergency medicine, such as cardiothoracic surgeons and pulmonologists, so everybody speaks the same 'diagnostic language'. Several educational programs on lung and cardiac ultrasonography have been developed which can serve as an example $(1,18)$. Furthermore, also medical education has to focus on POCUS. An impressive nine year investigation on effectiveness of POCUS education for medical students showed promising results (19), and medical students seem to be quick learners in POCUS (20).

Third, some important logistical steps must be undertaken. The legal aspect of LUS has to be addressed. The question on how much experience a physician must have to be able to perform POCUS independently is still not answered. Between 20 and 50 examinations is considered sufficient $(21,22)$. In addition, POCUS must be distinguished from conventional ultrasound, which is specialized ultrasound performed by radiologists, cardiologists or gynecologists who received standard certified training (23). Implementation of POCUS asks for clear peer agreements, and POCUS must not be considered as a replacement of specialized ultrasound. As imaging is often repeated, consensus on documentation must be reached regarding which images should be reviewed and how to store them in patient records. Also, more ultrasound devices need to be purchased. However, technical development is rapid, with devices getting smaller, significantly cheaper and displaying improved image quality, this will be a smaller problem for the near future.

Fourth, LUS comes with certain limitations. Its high accuracy can have a downside, as it also increases the identification of small and potentially less clinical relevant findings. Therefore, LUS findings always need to be combined with clinical symptoms, to prevent overtreatment. In those cases where LUS acquisition has its limitations, for example in terms of intrinsic restrictions like subcutaneous emphysema or large thoracic dressings, the use of other diagnostic modalities is warranted, such as CXR or CT.

Lastly, future research must focus on LUS (and POCUS) as a primary diagnostic tool and correlate its findings to patient outcome. In doing so, we also must define settings in which LUS and conventional diagnostics can complement each other.

In conclusion, the evidence regarding the value of LUS in the perioperative setting is mounting. We are confident that it should be the primary diagnostic tool for diagnosing postoperative pulmonary complication in patients after (cardiothoracic) surgery, simply because it is more accurate and provides more meaningful information than auscultation and CXR. However, it is important to realize that LUS and CXR, or even auscultation can be complementary in certain situations, such as subcutaneous emphysema.

\section{Acknowledgements}

None.

\section{Footnote}

Conflicts of Interest: The authors have no conflicts of interest to declare.

\section{References}

1. Touw HR, Tuinman P, Gelissen H, et al. Lung ultrasound: routine practice for the next generation of internists. Neth J Med 2015;73:100-7.

2. Lichtenstein DA, Mezière GA. Relevance of lung ultrasound in the diagnosis of acute respiratory failure: the BLUE protocol. Chest 2008;134:117-25.

3. Winkler MH, Touw HR, van de Ven PM, et al. Diagnostic Accuracy of Chest Radiograph, and When Concomitantly Studied Lung Ultrasound, in Critically Ill Patients With Respiratory Symptoms: A Systematic Review and MetaAnalysis. Crit Care Med 2018;46:e707-14. 
4. Smit JM, Raadsen R, Blans MJ, et al. Bedside ultrasound to detect central venous catheter misplacement and associated iatrogenic complications: a systematic review and metaanalysis. Critical Care 2018;22:65.

5. Zanobetti M, Coppa A, Bulletti F, et al. Verification of correct central venous catheter placement in the emergency department: comparison between ultrasonography and chest radiography. Intern Emerg Med 2013;8:173-80.

6. Steenvoorden TS, Smit JM, Haaksma ME, et al. Necessary additional steps in ultrasound guided central venous catheter placement: getting to the heart of the matter. Crit Care 2017;21:307.

7. Haaksma M, Atmowihardjo L, Heunks L, et al. Ultrasound imaging of the diaphragm: facts and future. A guide for the bedside clinician. Netherlands Journal Of Critical Care 2018;26:58-63.

8. Haaksma M, Tuinman PR, Heunks L. Ultrasound to assess diaphragmatic function in the critically ill-a critical perspective. Ann Transl Med 2017;5:114.

9. Canet J, Gallart L, Gomar C, et al. Prediction of postoperative pulmonary complications in a populationbased surgical cohort. Anesthesiology 2010;113:1338-50.

10. Goudie E, Bah I, Khereba M, et al. Prospective trial evaluating sonography after thoracic surgery in postoperative care and decision making. Eur J Cardiothorac Surg 2012;41:1025-30.

11. Vezzani A, Manca T, Brusasco C, et al. Diagnostic value of chest ultrasound after cardiac surgery: a comparison with chest X-ray and auscultation. J Cardiothorac Vasc Anesth 2014;28:1527-32.

12. Alsaddique A, Royse AG, Royse CF, et al. Repeated Monitoring With Transthoracic Echocardiography and Lung Ultrasound After Cardiac Surgery: Feasibility and Impact on Diagnosis. J Cardiothorac Vasc Anesth 2016;30:406-12.

13. Canty D, Ford J, Heiberg J, et al. Point-of-care diagnosis of perioperative lung pathology with lung ultrasound in cardiothoracic surgery-comparison with clinical examination and chest $\mathrm{x}$-ray. J Cardiothorac Vasc Anesth

Cite this article as: Nooitgedacht $\mathrm{J}$, Haaksma M, Touw HR, Tuinman PR. Perioperative care with an ultrasound device is as Michael Jordan with Scotty Pippen: at its best! J Thorac Dis 2018;10(12):6436-6441. doi: 10.21037/jtd.2018.12.82
2017;31:S44-5.

14. Touw HR, Parlevliet KL, Beerepoot M, et al. Lung ultrasound compared with chest $\mathrm{X}$-ray in diagnosing postoperative pulmonary complications following cardiothoracic surgery: a prospective observational study. Anaesthesia 2018;73:946-54.

15. Chiappetta M, Meacci E, Cesario A, et al. Postoperative chest ultrasound findings and effectiveness after thoracic surgery: A pilot study. Ultrasound Med Biol 2018;44:1960-7.

16. Kubodera T, Adachi YU, Hatano T, et al. Subcutaneous emphysema and ultrasound sonography. J Intensive Care 2013;1:8.

17. Verniquet A, Kakel R. Subcutaneous emphysema: ultrasound barrier. Can J Anaesth 2011;58:336-7.

18. Mahmood F, Bortman J, Amir R, et al. Training Surgical Residents for Ultrasound-Guided Assessment and Management of Unstable Patients. J Surg Educ 2018. [Epub ahead of print].

19. Hoppmann RA, Rao VV, Bell F, et al. The evolution of an integrated ultrasound curriculum (iUSC) for medical students: 9-year experience. Crit Ultrasound J 2015;7:18.

20. Andersen GN, Viset A, Mjølstad OC, et al. Feasibility and accuracy of point-of-care pocket-size ultrasonography performed by medical students. BMC Med Educ 2014;14:156.

21. Pietersen PI, Madsen KR, Graumann O, et al. Lung ultrasound training: a systematic review of published literature in clinical lung ultrasound training. Crit Ultrasound J 2018;10:23.

22. Galarza L, Wong A, Malbrain MLNG. The state of critical care ultrasound training in Europe: A survey of trainers and a comparison of available accreditation programmes. Anaesthesiol Intensive Ther 2017;49:382-6.

23. Elbers PWG, Kamp O, van der Sluijs JP, et al. Should every doctor be allowed to use ultrasound? Dilemmas surrounding broad application of ultrasound in clinical practice. Ned Tijdschr Geneeskd 2017;161:D1063. 